\title{
AN EXTENSION OF A LYAPUNOV APPROACH TO THE STABILIZATION OF SECOND ORDER COUPLED SYSTEMS ${ }^{*, * *, * * *}$
}

\author{
Thierry Horsin ${ }^{1, * * * *}$ And Mohamed Ali Jendoubi ${ }^{2,3}$
}

\begin{abstract}
This paper deals with the convergence to zero of the energy of the solutions of a second order linear coupled system. It revisits some previous results on the stabilization of such systems by exhibiting Lyapunov functions. The ones used are constructed according to some scalar cases situations. These simpler situations explicitely show that the assumptions made on the operators in the coupled systems seem, first, natural and, second, give insight on their forms.
\end{abstract}

Mathematics Subject Classification. 35B40, 49J15, 49J20.

Received March 27, 2019. Accepted November 27, 2019.

\section{INTRODUCTION, FUNCTIONAL FRAMEWORK}

Let us consider a quite general coupled system in abstract form

$$
\left\{\begin{array}{l}
u^{\prime \prime}+B u^{\prime}+A_{1} u+\alpha C v=0 \\
v^{\prime \prime}+A_{2} v+\alpha C^{*} u=0
\end{array}\right.
$$

where $A_{1}$ and $A_{2}$ and $C$ are, in general, unbounded operators. F. Alabau et al. considered in [4], the case when $C=I d$, and $A_{1}$ and $A_{2}$ are densely closed linear self-adjoint coercive operator and $B$ is a coercive bounded self-adjoint operator. They proved that if $|\alpha|\|C\|<1$ then the energy of the solution $(u, v)$ in polynomially decreasing under quite large assumption on $A_{1}$ and $A_{2}$. In this paper our main concern is the case $A_{2}=A_{1}^{2}$ which is a special case of the aforementioned paper.

When $A_{1}=A_{2}$ and $|\alpha|\|C\|<1$, A. Haraux and M.A. Jendoubi proved in [9] (see also [8]) the polynomial convergence to 0 of the energy by means of a Lyapunov method.

* The first author wishes to thank the Tunisian Mathematical Society (SMT) for its kind invitation to its annual congress during which this work was completed.

** The second author wishes to thank the department of mathematics and statistics EPN6 and the research department M2N (EA7340) of the CNAM where this work was initiated.

*** Both authors are grateful to the reviewers for their helpful comments and suggestions.

Keywords and phrases: damping, linear evolution equations, dissipative hyperbolic equation, decay rates, Lyapunov function.

${ }^{1}$ Laboratoire M2N, EA7340, CNAM, 292 rue Saint-Martin, 75003 Paris France.

${ }^{2}$ Université de Carthage, Institut Préparatoire aux Etudes Scientifiques et Techniques, B.P. 512070 La Marsa, Tunisia.

3 Laboratoire équations aux dérivées partielles, Faculté des sicences de Tunis, Université Tunis El Manar, Campus Universitaire El Manar, 2092 El Manar, Tunisia.

**** Corresponding author: thierry.horsin@lecnam.net 
As we previously said, in this paper, we investigate such a method in the case when $A_{2}=A_{1}^{2}$ and $C=A_{1}^{\beta}$ with $\beta \in\left[0, \frac{3}{2}\right]$. The main result of this paper is Theorem 3.3 which also proves the polynomial convergence to 0 of the solution $(u, v)$. Compared to the result in ([4], p. 144, Prop. 5.3), the convergence that we obtain is in weaker norms, but requires less regularity on the initial data.

Let us mention that the stabilization of such systems settled in abstract form has been widely studied and many results are connected to those in our paper. The case when $A_{1}$ and $A_{2}$ are unbounded operators, and $C$ is a bounded was considered by Alabau in [3] and by Alabau et al. in [5] where the polynomial statibility is proven. The optimal energy decay was proven in [11] in the case $A_{1}=A_{2}=A, C=I$ and $B=A^{\gamma}$ where $\gamma<0$. The indirect stabilization of abstract coupled equations is also considered in $[1,2,6,7]$.

Nonlinear damping are also currently investigated. See e.g. [12].

In order to motivate the Lyapunov function that we construct in the proof of our main result, we explain the strategy in Section 2 in the framework of a coupled scalar differential system.

In Section 3 we introduce the functional framework and an existence theorem that lead to state and prove our main result, namely Theorem 3.3.

\section{A Lyapunov FunCtion FOR the SCALAR CASE}

As mentioned in the preceding section, we consider the (real) scalar coupled system

$$
\left\{\begin{array}{l}
u^{\prime \prime}+u^{\prime}+\lambda u+c v=0 \\
v^{\prime \prime}+\mu v+c u=0
\end{array}\right.
$$

where $\lambda, \mu>0$, and $c$ are such that $0<c^{2}<\lambda \mu$. The damping coefficient is set to 1 for simplicity but a time scale change reduces general damping terms $b u^{\prime}$ to this case. In order to shorten the formulas, let us introduce for each solution $(u, v)$ of $(2.1)$, its total energy

$$
\mathcal{E}\left(u, u^{\prime}, v, v^{\prime}\right)=\frac{1}{2}\left[u^{\prime 2}+v^{\prime 2}+\lambda u^{2}+\mu v^{2}\right]+c u v .
$$

Then we have for all $t \geq 0$

$$
\frac{d}{d t} \mathcal{E}\left(u, u^{\prime}, v, v^{\prime}\right)=-u^{\prime 2}
$$

Now we introduce

$$
K(t)=\frac{1}{2}\left[u^{\prime 2}+v^{\prime 2}+\lambda u^{2}+\mu v^{2}\right] .
$$

Our first result is the following

Proposition 2.1. There are some constants $\eta>0, \delta>0$ such that

$$
\forall t \geq 0 \quad K(t) \leq \eta e^{-\delta t} K(0) .
$$

Proof. For all $\varepsilon>0$ we define the function

$$
H_{\varepsilon}=\mathcal{E}-\varepsilon v v^{\prime}+2 \varepsilon u u^{\prime}+\frac{3 \varepsilon}{2 c}\left(\mu u^{\prime} v-\lambda u v^{\prime}\right) .
$$


It is easy to check that

$$
C_{1} K(t) \leq H_{\varepsilon}(t) \leq C_{2} K(t)
$$

where

$$
C_{1}=\left[\frac{\sqrt{\lambda \mu}-|c|}{\sqrt{\lambda \mu}}-\varepsilon\left(\frac{2}{\min (\sqrt{\lambda}, \sqrt{\mu})}+\frac{3}{2|c|} \max (\sqrt{\lambda}, \sqrt{\mu})\right)\right]
$$

and

$$
C_{2}=\left[\frac{\sqrt{\lambda \mu}+|c|}{\sqrt{\lambda \mu}}+\varepsilon\left(\frac{2}{\min (\sqrt{\lambda}, \sqrt{\mu})}+\frac{3}{2|c|} \max (\sqrt{\lambda}, \sqrt{\mu})\right)\right]
$$

In fact, using Young's inequality, we get

$$
\begin{aligned}
|c u v| & =\frac{c}{\sqrt{\lambda} \sqrt{\mu}} \sqrt{\lambda}|u| \sqrt{\mu}|v| \leq \frac{|c|}{\sqrt{\lambda} \sqrt{\mu}}\left[\frac{\lambda}{2} u^{2}+\frac{\mu}{2} v^{2}\right] \\
\left|-\varepsilon v v^{\prime}\right| & =\frac{\varepsilon}{\sqrt{\mu}} \sqrt{\mu}|v|\left|v^{\prime}\right| \leq \frac{\varepsilon}{\sqrt{\mu}}\left[\frac{\mu}{2} v^{2}+\frac{v^{\prime 2}}{2}\right] \\
\left|2 \varepsilon u u^{\prime}\right| & =\frac{2 \varepsilon}{\sqrt{\lambda}} \sqrt{\lambda}|u|\left|u^{\prime}\right| \leq \frac{2 \varepsilon}{\sqrt{\lambda}}\left[\frac{\lambda}{2} u^{2}+\frac{u^{\prime 2}}{2}\right] \\
\left|\frac{3 \varepsilon}{2 c} \mu u^{\prime} v\right| & =\frac{3 \varepsilon}{2|c|} \sqrt{\mu}\left|u^{\prime}\right| \sqrt{\mu}|v| \leq \frac{3 \varepsilon}{2|c|} \sqrt{\mu}\left[\frac{u^{\prime 2}}{2}+\frac{\mu}{2} v^{2}\right] \\
\left|-\frac{3 \varepsilon}{2|c|} \lambda u v^{\prime}\right| & =\frac{3 \varepsilon}{2|c|} \sqrt{\lambda} \sqrt{\lambda} u v^{\prime} \leq \frac{3 \varepsilon}{2|c|} \sqrt{\lambda}\left[\lambda \frac{u^{2}}{2}+\frac{v^{\prime 2}}{2}\right]
\end{aligned}
$$

Then we deduce

$$
\begin{aligned}
& H_{\varepsilon} \leq \underbrace{\left[1+\varepsilon\left(\frac{2}{\sqrt{\lambda}}+\frac{3 \sqrt{\mu}}{2|c|}\right)\right]}_{A_{1}} \frac{u^{\prime 2}}{2}+\underbrace{\left[1+\varepsilon\left(\frac{1}{\sqrt{\mu}}+\frac{3 \sqrt{\lambda}}{2|c|}\right)\right]}_{A_{2}} \frac{v^{\prime 2}}{2}+ \\
& \underbrace{\left[1+\frac{|c|}{\sqrt{\lambda} \sqrt{\mu}}+\varepsilon\left(\frac{2}{\sqrt{\lambda}}+\frac{3 \sqrt{\lambda}}{2|c|}\right)\right]}_{A_{3}} \frac{\lambda}{2} u^{2}+\underbrace{\left[1+\frac{|c|}{\sqrt{\lambda} \sqrt{\mu}}+\varepsilon\left(\frac{1}{\sqrt{\mu}}+\frac{3 \sqrt{\lambda}}{2|c|}\right)\right]}_{A_{4}} \frac{\mu}{2} v^{2} .
\end{aligned}
$$

It is clear that for all $i \in\{1,2,3,4\}$

$$
A_{i} \leq C_{2}
$$

where $C_{2}$ is given in (2.5). A similar proof gives similar inequalities for $C_{1}$.

Let $\varepsilon_{1}>0$ the value of $\varepsilon$ such that $C_{1}$ defined in (2.4) is equal to 0 . Let $\varepsilon \in\left(0, \varepsilon_{1}\right)$. In this case $C_{1}$ becomes positive. 
A straightforward computation gives

$$
\begin{aligned}
H_{\varepsilon}^{\prime}=- & u^{\prime 2}-\varepsilon v^{\prime 2}-\varepsilon v v^{\prime \prime}+2 \varepsilon u^{\prime 2}+2 \varepsilon u u^{\prime \prime}+\frac{3 \varepsilon}{2 c}\left[(\mu-\lambda) u^{\prime} v^{\prime}+\mu u^{\prime \prime} v-\lambda u v^{\prime \prime}\right] \\
=- & (1-2 \varepsilon) u^{\prime 2}-\varepsilon v^{\prime 2}+\varepsilon c u v+\varepsilon \mu v^{2}-2 \varepsilon u u^{\prime}-p \varepsilon \lambda u^{2}-2 \varepsilon c u v \\
& +\frac{3 \varepsilon}{2 c}\left[(\mu-\lambda) u^{\prime} v^{\prime}-\mu u^{\prime} v-\mu \lambda u v-\mu c v^{2}+\lambda c u^{2}+\lambda \mu u v\right] \\
=- & (1-2 \varepsilon) u^{\prime 2}-\varepsilon v^{\prime 2}-\lambda \varepsilon \frac{1}{2} u^{2}-\mu \varepsilon \frac{1}{2} v^{2}-2 \varepsilon u u^{\prime}-\varepsilon c u v \\
& \quad-\mu \varepsilon \frac{3}{2 c} u^{\prime} v+(\mu-\lambda) \varepsilon \frac{3}{2 c} u^{\prime} v^{\prime} .
\end{aligned}
$$

Now we have

$$
\begin{aligned}
\lambda u^{2}+2 c u v+\mu v^{2} & =\lambda\left(u^{2}+\frac{2 c}{\lambda} u v+\frac{\mu}{\lambda} v^{2}\right) \\
& =\lambda\left[\left(u+\frac{c}{\lambda} v\right)^{2}+\left(\frac{\mu}{\lambda}-\frac{c^{2}}{\lambda^{2}}\right) v^{2}\right] \\
& \geq \frac{\lambda \mu-c^{2}}{\lambda} v^{2} .
\end{aligned}
$$

Similarly, we get

$$
\lambda u^{2}+2 c u v+\mu v^{2} \geq \frac{\lambda \mu-c^{2}}{\mu} u^{2}
$$

and then

$$
\lambda u^{2}+2 c u v+\mu v^{2} \geq \frac{\lambda \mu-c^{2}}{2}\left(\frac{1}{\mu} u^{2}+\frac{1}{\lambda} v^{2}\right)
$$

Using Young's inequality, we can find some constants $c_{1}, c_{2}, c_{3}>0$ such that

$$
\begin{aligned}
& \left|2 u u^{\prime}\right| \leq \frac{1}{8 \mu}\left(\lambda \mu-c^{2}\right) u^{2}+c_{1} u^{\prime 2} ; \\
& \frac{3}{2 c}\left|\mu u^{\prime} v\right| \leq \frac{1}{8 \lambda}\left(\lambda \mu-c^{2}\right) v^{2}+c_{2} u^{\prime 2} ; \\
& \frac{3}{2 c}|\mu-\lambda|\left|u^{\prime} v^{\prime}\right| \leq \frac{1}{2} v^{\prime 2}+c_{3} u^{\prime 2} .
\end{aligned}
$$

Finally we obtain

$$
H_{\varepsilon}^{\prime} \leq-\left(1-\left(2+c_{1}+c_{2}+c_{3}\right) \varepsilon\right) u^{\prime 2}-\frac{\varepsilon}{2} v^{\prime 2}-\frac{\varepsilon}{8 \mu}\left(\lambda \mu-c^{2}\right) u^{2}-\frac{\varepsilon}{8 \mu}\left(\lambda \mu-c^{2}\right) v^{2} .
$$

Now by choosing $\varepsilon \in\left(0, \varepsilon_{1}\right)$ such that $1-\left(2+c_{1}+c_{2}+c_{3}\right) \varepsilon>0$, some constant $C_{3}>0$ can be found such that

$$
H_{\varepsilon}^{\prime} \leq-C_{3} K(t)
$$


By combining this with the inequality (2.3), we get for all $t \geq 0$

$$
H_{\varepsilon}^{\prime}(t) \leq-\frac{C_{3}}{C_{2}} H_{\varepsilon}(t)
$$

We conclude the proof by integrating this last inequality and using (2.3) again.

$$
\text { 3. The CASE } A_{2}=A^{2} \text {, AND } C=A^{\beta} \text { With } \beta \in\left[0, \frac{3}{2}\right]
$$

This section is devoted to the proof of Theorem 3.3. In order to proceed we first introduce the functional framework and give an existence theorem.

\subsection{Functional framework}

Let $H$ be a separable Hilbert space, whose norm and scalar product will be denoted $\|\cdot\|$ and $\langle\cdot, \cdot\rangle$ respectively. We consider $A: H \rightarrow H$ an unbounded closed self-adjoint operator such that the injection $D(A) \subset H$ is dense and compact. We assume moreover throughout the paper that there exists $a>0$ such that

$$
\forall u \in D(A), \quad\langle A u, u\rangle \geq a\langle u, u\rangle .
$$

Following for example the exposition given in [10], by denoting $\left(\lambda_{n}\right)_{n \in \mathbb{N}^{*}}$ the increasing sequence of eigenvalues of $A$, the largest $a$ for which (3.1) is true is $\lambda_{1}$.

Besides, let us consider $\left(e_{n}\right)_{n \in \mathbb{N}^{*}}$ an orthonormal basis of $H$ constituted by eigenvectors of $A$. For any $\beta>0$, we consider $u=\sum_{n=1}^{\infty}\left\langle u, e_{i}\right\rangle e_{i}, \in H \quad\left(u \in H \Longleftrightarrow \sum_{n=1}^{\infty}\left\langle u, e_{i}\right\rangle^{2}<\infty\right)$, and we set $A^{\beta}: H \rightarrow H$ given by

$$
A^{\beta} u=\sum_{i=1}^{\infty} \lambda_{i}^{\beta}\left\langle u, e_{i}\right\rangle e_{i}
$$

then (see e.g. [10])

$$
D\left(A^{\beta}\right)=\left\{u \in H, \sum_{i=1}^{\infty} \lambda_{i}^{2 \beta}\left\langle u, e_{i}\right\rangle^{2}<\infty\right\}
$$

and $A^{\beta}$ is an unbounded self-adjoint operator such that the inclusion $D\left(A^{\beta}\right) \subset H$ is dense and compact. We also have

$$
\forall u \in D\left(A^{\beta}\right), \quad\left\langle A^{\beta} u, u\right\rangle \geq a\langle u, u\rangle
$$

for some $a>0$. The largest $a$ for which this inequality is true being $\lambda_{1}^{\beta}$.

As usual we write $A^{0}=\mathrm{Id}$. In this case of course the operator $A^{\alpha}$ is a continuous linear operator on $H$.

We will denote $V=D\left(A^{1 / 2}\right)$ and $W=D(A)$. Thus $V$ and $W$ are Hilbert spaces whose norms $\|\cdot\|_{V}$ and $\|\cdot\|_{W}$ are given respectively by

$$
\|u\|_{V}=\left\|A^{1 / 2} u\right\|, \quad\|u\|_{W}=\|A u\| .
$$

We have, if we identify $H$ with its dual

$$
W \subset V \subset H \subset V^{\prime} \subset W^{\prime}
$$


with dense and compact injections when the norms on the Hilbert spaces $V^{\prime}$ and $W^{\prime}$ are given by

$$
\forall u \in V^{\prime}, \quad\|u\|_{V^{\prime}}=\left\langle u, A^{-1} u\right\rangle_{V^{\prime}, V}^{1 / 2}
$$

and

$$
\forall u \in W^{\prime}, \quad\|u\|_{W^{\prime}}=\left\langle u, A^{-2} u\right\rangle_{W^{\prime}, W}^{1 / 2}
$$

where $\langle\cdot, \cdot\rangle_{V^{\prime}, V}$ denotes the action of $V^{\prime}$ on $V$ (with a similar notation for $W$ ). Of course when $u \in H$ one has

$$
\|u\|_{V^{\prime}}=\left\langle A^{-1} u, u\right\rangle^{1 / 2},\|u\|_{W^{\prime}}=\left\langle A^{-2} u, u\right\rangle^{1 / 2} .
$$

Let us remark that with these definitions $A$ maps continuously $V$ to $V^{\prime}$ and $A^{2}$ maps $W$ to $W^{\prime}$.

\subsection{Existence result}

Let $\alpha$ and $\beta$ two reals numbers with $\beta \geq 0$. We recall that we consider the problem

$$
\left\{\begin{array}{l}
u^{\prime \prime}+u^{\prime}+A u+\alpha A^{\beta} v=0 \\
v^{\prime \prime}+A^{2} v+\alpha A^{\beta} u=0
\end{array}\right.
$$

which can be rewritten as the first order system

$$
\left\{\begin{array}{l}
u^{\prime}-w=0 \\
v^{\prime}-z=0 \\
w^{\prime}+A u+w+\alpha A^{\beta} v=0 \\
z^{\prime}+A^{2} v+\alpha A^{\beta} u=0 .
\end{array}\right.
$$

Let us first establish an existence and uniqueness result for (3.5).

We concentrate on the case $\beta \in\left[\frac{1}{2}, \frac{3}{2}\right]$, the case $\beta \in\left[0, \frac{1}{2}\right)$ being easier.

Let us consider

$$
\mathcal{H}:=V \times W \times H \times H .
$$

For two elements of $\mathcal{H} U_{i}=\left(u_{i}, v_{i}, w_{i}, z_{i}\right), i=1,2$, we define

$$
\begin{aligned}
\left\langle U_{1}, U_{2}\right\rangle_{\mathcal{H}}:= & \left\langle A u_{2}, u_{1}\right\rangle_{V^{\prime}, V}+\left\langle A^{2} v_{2}, v_{1}\right\rangle_{W^{\prime}, W}+\left\langle w_{1}, w_{2}\right\rangle+\left\langle z_{1}, z_{2}\right\rangle \\
& +\alpha\left\langle A^{\beta} v_{2}, u_{1}\right\rangle_{V^{\prime}, V}+\alpha\left\langle A^{\beta} u_{2}, v_{1}\right\rangle_{W^{\prime}, W},
\end{aligned}
$$

where $\langle\cdot, \cdot\rangle_{V^{\prime}, V}$ denotes the usual duality pairing between $V$ and $V^{\prime}$, with similar notation for $W$, while $\langle\cdot, \cdot\rangle$ denotes the scalar product on $H$ for which it is a Hilbert space.

It is straightforward to prove that $\langle\cdot, \cdot\rangle_{\mathcal{H}}$ defines a scalar product on $\mathcal{H}$ for which it is a Hilbert space provided that

$$
|\alpha|<\lambda_{1}^{\frac{3-2 \beta}{2}}
$$

From now on we assume that (3.7) holds true. 
We now consider the unbounded operator $\mathcal{A}: \mathcal{H} \longrightarrow \mathcal{H}$ defined by

$$
D(\mathcal{A}):=\left\{U=(u, v, w, z) \in \mathcal{H},\left(-w,-z, A u+\alpha A^{\beta} v+w, A^{2} v+\alpha A^{\beta} u\right) \in \mathcal{H}\right\}
$$

and for $U=(u, v, w, z) \in D(\mathcal{A})$

$$
\mathcal{A} U=\left(-w,-z, A u+\alpha A^{\beta} v+w, A^{2} v+\alpha A^{\beta} u\right) .
$$

It is clear that $\mathcal{A}$ has a dense domain in $\mathcal{H}$.

Let us remark that for any $U=(u, v, w, z) \in D(\mathcal{A})$ one has

$$
\begin{aligned}
\langle\mathcal{A} U, U\rangle_{\mathcal{H}}= & -\langle A u, w\rangle_{V^{\prime}, V}-\left\langle A^{2} v, z\right\rangle_{W^{\prime}, W}+\left\langle A u+\alpha A^{\beta} v+w, w\right\rangle+\left\langle A^{2} v+\alpha A^{\beta} u, z\right\rangle \\
& -\alpha\left\langle A^{\beta} v, w\right\rangle_{V^{\prime}, V}-\alpha\left\langle A^{\beta} u, z\right\rangle_{W^{\prime}, W}
\end{aligned}
$$

and therefore $\langle\mathcal{A} U, U\rangle=\|w\|^{2} \geq 0$. Indeed

$$
\left\langle A^{2} v+\alpha A^{\beta} u, z\right\rangle=\left\langle A^{2} v+\alpha A^{\beta} u, z\right\rangle_{W^{\prime}, W},
$$

since $A^{\beta} u \in W^{\prime}$ for $\beta \in[1,3 / 2]$ and $u \in V=D\left(A^{1 / 2}\right)$.

Let us show that $I+\mathcal{A}$ is onto. For this we take $(f, g, h, k) \in \mathcal{H}$. We want to find $(u, v, w, z) \in D(\mathcal{A})$ such that

$$
\begin{aligned}
& u-w=f \\
& v-z=g \\
& A u+\alpha A^{\beta} v+2 u=h+2 f \\
& A^{2}+\alpha A^{\beta} u+v=k+g .
\end{aligned}
$$

We define $\Phi:(V \times W)^{2} \rightarrow \mathbb{R}$ by

$$
\begin{aligned}
\Phi\left(u_{1}, v_{1}, u_{2}, v_{2}\right):= & \left\langle A^{1 / 2} u_{1}, A^{1 / 2} u_{2}\right\rangle+\left\langle A v_{1}, A v_{2}\right\rangle+\alpha\left\langle A^{\beta} v_{1}, u_{2}\right\rangle_{V^{\prime}, V}+ \\
& \alpha\left\langle A u_{1}, A^{\beta-1} v_{2}\right\rangle_{V^{\prime}, V}+2\left\langle u_{1}, u_{2}\right\rangle+\left\langle v_{1}, v_{2}\right\rangle .
\end{aligned}
$$

Clearly $\Phi$ is continuous on $V \times W$. It is also clear that $\Phi$ is coercive if we assume $|\alpha|<\lambda_{1}^{\frac{3-2 \beta}{2}}$.

By the Lax-Milgram theorem, there exists a unique $(u, v) \in V \times W$ such that

$$
\forall(\delta u, \delta v) \in V \times W, \quad \Phi(u, v, \delta u, \delta v)=\langle h+2 f, \delta u\rangle+\langle k+g, \delta v\rangle .
$$

We therefore get

$$
\begin{gathered}
A^{2} v+\alpha A^{\beta} u+v=g+k \\
A u+\alpha A^{\beta} v+2 u=h+2 f .
\end{gathered}
$$

Now if we denote $w=u-f$ and $z=v-g$ then $w \in V$ since $u, f \in V$ and $z \in W$ since $v, g \in W$.

We have thus proven that $\mathcal{A}$ is maximal monotone. By classical theory, we get that

Theorem 3.1. For any $\left(u_{0}, v_{0}, u_{1}, v_{1}\right) \in \mathcal{H}$, there exists a unique solution to $(3.5)$ in $C([0, T], \mathcal{H}) \times$ $C^{1}\left([0, T], D(\mathcal{A})^{\prime}\right)$. 
Remark 3.2. It is also well known that if $\left(u_{0}, v_{0}, u_{1}, v_{1}\right) \in D(\mathcal{A})$ then the solution to (3.5) belongs to $C([0, T], D(\mathcal{A})) \cap C^{1}(0, T, \mathcal{H})$.

\subsection{Main result of the paper}

Our main result is the following

Theorem 3.3. Assume $\beta \in\left[0, \frac{3}{2}\right]$ and $\alpha \neq 0$. Let $(u, v)$ be a solution of (3.5) such that $\left(u(0), u^{\prime}(0), v(0), v^{\prime}(0)\right) \in$ $\mathcal{H}$, then there exists a constant $c>0$ such that

$$
\begin{array}{cl}
\forall t>0, & \left\|A^{\frac{\beta}{2}-1} u^{\prime}(t)\right\|_{W^{\prime}}^{2}+\left\|A^{\frac{\beta}{2}-1} v^{\prime}(t)\right\|_{W^{\prime}}^{2}+\left\|A^{\frac{\beta-1}{2}} u(t)\right\|_{W^{\prime}}^{2}+\left\|A^{\frac{\beta}{2}} v(t)\right\|_{W^{\prime}}^{2} \\
& \leq \frac{c}{t}\left[\left\|u^{\prime}(0)\right\|^{2}+\left\|v^{\prime}(0)\right\|^{2}+\|u(0)\|_{V}^{2}+\|v(0)\|_{W}^{2}\right] \quad \text { if } \beta \in[0,1] ; \\
\forall t>0, & \left\|A^{\frac{-\beta}{2}} u^{\prime}(t)\right\|_{W^{\prime}}^{2}+\left\|A^{\frac{-\beta}{2}} v^{\prime}(t)\right\|_{W^{\prime}}^{2}+\left\|A^{\frac{1-\beta}{2}} u(t)\right\|_{W^{\prime}}^{2}+\left\|A^{1-\frac{\beta}{2}} v(t)\right\|_{W^{\prime}}^{2} \\
& \leq \frac{c}{t}\left[\left\|u^{\prime}(0)\right\|^{2}+\left\|v^{\prime}(0)\right\|^{2}+\|u(0)\|_{V}^{2}+\|v(0)\|_{W}^{2}\right] \text { if } \beta \in\left[1, \frac{3}{2}\right] .
\end{array}
$$

Remark 3.4. If we replace (3.5) by

$$
\left\{\begin{array}{l}
u^{\prime \prime}+B u^{\prime}+A u+\alpha A^{\beta} v=0 \\
v^{\prime \prime}+A^{2} v+\alpha A^{\beta} u=0
\end{array}\right.
$$

where, as mentionned in the introduction, $B$ is a bounded self-adjoint operator on $H$ for which there exists $\mu>0$ such that

$$
\langle B u, u\rangle \geq \mu\|u\|^{2}
$$

the results of Theorem 3.1 and Remark 3.4 remain true.

Remark 3.5. If we replace (3.5) by

$$
\left\{\begin{array}{l}
u^{\prime \prime}+B u^{\prime}+A u+\alpha A^{\beta} v=0 \\
v^{\prime \prime}+A_{2} v+\alpha A^{\beta} u=0
\end{array}\right.
$$

where $A_{2}$ is a self-adjoint unbounded operator such that $D\left(A_{2}\right)=D\left(A^{2}\right)$ and there exist $\nu_{1}, \nu_{2}>0$ such that

$$
\forall u \in D\left(A_{2}\right), \nu_{1}\left\langle A^{2} u, u\right\rangle \leq\left\langle A_{2} u, u\right\rangle \leq \nu_{2}\left\langle A^{2} u, u\right\rangle
$$

and if $B$ is as in the remark 3.4, the result of Theorem 3.3 remains true provided $|\alpha|$ is small enough (depending on $\lambda_{1}, \nu_{1}$ and $\nu_{2}$ ).

Remark 3.6. In the case $\beta=0$, in order to obtain the decay of the energy, we must assume

$$
A^{2} u(0) \in V, \quad A^{2} v(0) \in W, \quad A^{2} u^{\prime}(0) \in H, \quad A^{2} v^{\prime}(0) \in H .
$$

In the paper [4], the authors obtain such a decay with merely

$$
A u(0) \in V, \quad A^{2} v(0) \in W, \quad A u^{\prime}(0) \in H, \quad A^{2} v^{\prime}(0) \in H .
$$


We, of course, would expect that the energy decay also holds with

$$
\left(u(0), v(0), u^{\prime}(0), v^{\prime}(0)\right) \in D(\mathcal{A})
$$

but unfortunately we are not able to prove it for the moment being.

Proof of Theorem 3.3. All the computations below will be made assuming that $\left(u_{0}, v_{0}, u_{1}, v_{1}\right) \in D(\mathcal{A})$ which ascertains them. By density and continuity the inequalities stated in Theorem 3.3 remain true.

Let us also recall that $\alpha$ satisfies $|\alpha|<\lambda_{1}^{\frac{3-2 \beta}{2}}$.

We introduce the energy of the system by

$$
E(t)=\frac{1}{2}\left[\left\|u^{\prime}(t)\right\|^{2}+\left\|v^{\prime}(t)\right\|^{2}+\|u(t)\|_{V}^{2}+\|v(t)\|_{W}^{2}\right]+\alpha\left\langle A^{\beta} v, u\right\rangle .
$$

Then we have

$$
E^{\prime}(t)=-\left\|u^{\prime}(t)\right\|^{2}
$$

Let $p>1$ and $\varepsilon>0$ two real numbers to be fixed later and let

$$
H_{\varepsilon}=E-\varepsilon \lambda_{1}^{2-\beta}\left\langle A^{\beta-2} v, v^{\prime}\right\rangle_{W^{\prime}}+p \varepsilon \lambda_{1}^{-a}\left\langle A^{a} u, u^{\prime}\right\rangle_{W^{\prime}}+\rho \varepsilon\left[\left\langle u^{\prime}, v\right\rangle_{W^{\prime}}-\left\langle u, A^{-1} v^{\prime}\right\rangle_{W^{\prime}}\right]
$$

where $\rho=\frac{p+1}{2 \alpha} \lambda_{1}^{2-\beta}$ and $a=\min (0,1-\beta)$. We find easily

$$
\begin{aligned}
H_{\varepsilon}^{\prime}= & -\left\|u^{\prime}\right\|^{2}-\varepsilon \lambda_{1}^{2-\beta}\left\|A^{\frac{\beta}{2}-1} v^{\prime}\right\|_{W^{\prime}}^{2}-\varepsilon \lambda_{1}^{2-\beta}\left\langle A^{\beta-2} v, v^{\prime \prime}\right\rangle_{W^{\prime}}+p \varepsilon \lambda_{1}^{-a}\left\|A^{\frac{a}{2}} u^{\prime}\right\|_{W^{\prime}}^{2} \\
& -p \varepsilon \lambda_{1}^{-a}\left\langle A^{a} u, u^{\prime}+A u+\alpha A^{\beta} v\right\rangle_{W^{\prime}}+\rho \varepsilon\left\langle u^{\prime}, v^{\prime}\right\rangle_{W^{\prime}}-\rho \varepsilon\left\langle u^{\prime}, A^{-1} v^{\prime}\right\rangle_{W^{\prime}} \\
& -\rho \varepsilon\left\langle u^{\prime}+A u+\alpha A^{\beta} v, v\right\rangle_{W^{\prime}}+\rho \varepsilon\left\langle u, A^{-1}\left(A^{2} v+\alpha A^{\beta} u\right)\right\rangle_{W^{\prime}} \\
= & -\left\|u^{\prime}\right\|^{2}-\varepsilon \lambda_{1}^{2-\beta}\left\|A^{\frac{\beta}{2}-1} v^{\prime}\right\|_{W^{\prime}}^{2}+\varepsilon \lambda_{1}^{2-\beta}\left\langle A^{\beta-2} v, A^{2} v+\alpha A^{\beta} u\right\rangle_{W^{\prime}}+p \varepsilon \lambda_{1}^{-a}\left\|A^{\frac{a}{2}} u^{\prime}\right\|_{W^{\prime}}^{2} \\
& -p \varepsilon \lambda_{1}^{-a}\left\langle A^{a} u, u^{\prime}\right\rangle_{W^{\prime}}-p \varepsilon \lambda_{1}^{-a}\left\|A^{\frac{a+1}{2}} u\right\|_{W^{\prime}}^{2}-p \varepsilon \lambda_{1}^{-a} \alpha\left\langle A^{a} u, A^{\beta} v\right\rangle_{W^{\prime}}+\rho \varepsilon\left\langle u^{\prime}, v^{\prime}\right\rangle_{W^{\prime}} \\
& -\rho \varepsilon\left\langle u^{\prime}, A^{-1} v^{\prime}\right\rangle_{W^{\prime}}-\rho \varepsilon\left\langle u^{\prime}, v\right\rangle_{W^{\prime}}-\rho \varepsilon \alpha\left\|A^{\frac{\beta}{2}} v\right\|_{W^{\prime}}+\rho \varepsilon \alpha\left\|A^{\frac{\beta-1}{2}} u\right\|_{W^{\prime}}^{2} \\
= & -\left\|u^{\prime}\right\|^{2}-\varepsilon \lambda_{1}^{2-\beta}\left\|A^{\frac{\beta}{2}-1} v^{\prime}\right\|_{W^{\prime}}^{2}+\varepsilon \lambda_{1}^{2-\beta}\left\|A^{\frac{\beta}{2}} v\right\|_{W^{\prime}}^{2}+\varepsilon \lambda_{1}^{2-\beta} \alpha\left\langle A^{\beta-2} v, A^{\beta} u\right\rangle_{W^{\prime}}+p \varepsilon \lambda_{1}^{-a}\left\|A^{\frac{a}{2}} u^{\prime}\right\|_{W^{\prime}}^{2} \\
& -p \varepsilon \lambda_{1}^{-a}\left\langle A^{a} u, u^{\prime}\right\rangle_{W^{\prime}}-p \varepsilon \lambda_{1}^{-a}\left\|A^{\frac{a+1}{2}} u\right\|_{W^{\prime}}^{2}-p \varepsilon \lambda_{1}^{-a} \alpha\left\langle A^{a} u, A^{\beta} v\right\rangle_{W^{\prime}}+\rho \varepsilon\left\langle u^{\prime}, v^{\prime}\right\rangle_{W^{\prime}} \\
& -\rho \varepsilon\left\langle u^{\prime}, A^{-1} v^{\prime}\right\rangle_{W^{\prime}}-\rho \varepsilon\left\langle u^{\prime}, v\right\rangle_{W^{\prime}}-\rho \varepsilon \alpha\left\|^{\frac{\beta}{2}} v\right\|_{W^{\prime}}^{2}+\rho \varepsilon \alpha\left\|A^{\frac{\beta-1}{2}} u\right\|_{W^{\prime}}^{2} \\
= & -\left\|u^{\prime}\right\|^{2}+p \varepsilon \lambda_{1}^{-a}\left\|A^{\frac{a}{2}} u^{\prime}\right\|_{W^{\prime}}^{2}-\varepsilon \lambda_{1}^{2-\beta}\left\|A^{\frac{\beta}{2}-1} v^{\prime}\right\|_{W^{\prime}}^{2}-p \varepsilon \lambda_{1}^{-a}\left\|A^{\frac{a+1}{2}} u\right\|_{W^{\prime}}^{2}-\varepsilon \frac{p-1}{2} \lambda_{1}^{2-\beta}\left\|A^{\frac{\beta}{2}} v\right\|_{W^{\prime}}^{2} \\
& +\rho \varepsilon \alpha\left\|A^{\frac{\beta-1}{2}} u\right\|_{W^{\prime}}^{2}+\varepsilon \lambda_{1}^{2-\beta} \alpha\left\langle A^{\beta-2} v, A^{\beta} u\right\rangle_{W^{\prime}}-p \varepsilon \lambda_{1}^{-a}\left\langle A^{a} u, u^{\prime}\right\rangle_{W^{\prime}}-p \varepsilon \lambda_{1}^{-a} \alpha\left\langle A^{a} u, A^{\beta} v\right\rangle_{W^{\prime}} \\
& +\rho \varepsilon\left\langle u^{\prime}, v^{\prime}\right\rangle_{W^{\prime}}-\rho \varepsilon\left\langle u^{\prime}, A^{-1} v^{\prime}\right\rangle_{W^{\prime}}-\rho \varepsilon\left\langle u^{\prime}, v\right\rangle_{W^{\prime}} .
\end{aligned}
$$

First case $: \beta \in[0,1]$. In this case $a=0$. We have

$$
\begin{aligned}
H_{\varepsilon}^{\prime}= & -\left\|u^{\prime}\right\|^{2}+p \varepsilon\left\|u^{\prime}\right\|_{W^{\prime}}^{2}-\varepsilon \lambda_{1}^{2-\beta}\left\|A^{\frac{\beta}{2}-1} v^{\prime}\right\|_{W^{\prime}}^{2}-p \varepsilon\left\|A^{\frac{1}{2}} u\right\|_{W^{\prime}}^{2}-\varepsilon \frac{p-1}{2} \lambda_{1}^{2-\beta}\left\|A^{\frac{\beta}{2}} v\right\|_{W^{\prime}}^{2} \\
& +\rho \varepsilon \alpha\left\|A^{\frac{\beta-1}{2}} u\right\|_{W^{\prime}}^{2}+\varepsilon \lambda_{1}^{2-\beta} \alpha\left\langle A^{\beta-2} v, A^{\beta} u\right\rangle_{W^{\prime}}-p \varepsilon\left\langle u, u^{\prime}\right\rangle_{W^{\prime}}-p \varepsilon \alpha\left\langle u, A^{\beta} v\right\rangle_{W^{\prime}} \\
& +\rho \varepsilon\left\langle u^{\prime}, v^{\prime}\right\rangle_{W^{\prime}}-\rho \varepsilon\left\langle u^{\prime}, A^{-1} v^{\prime}\right\rangle_{W^{\prime}}-\rho \varepsilon\left\langle u^{\prime}, v\right\rangle_{W^{\prime}}
\end{aligned}
$$


Now since

$$
\left\|A^{\frac{\beta-1}{2}} u\right\|_{W^{\prime}}^{2} \leq \frac{1}{\lambda_{1}^{2-\beta}}\langle A u, u\rangle_{W^{\prime}}=\frac{1}{\lambda_{1}^{2-\beta}}\left\|A^{\frac{1}{2}} u\right\|_{W^{\prime}}^{2}
$$

we get

$$
\begin{aligned}
H_{\varepsilon}^{\prime} \leq & -\left\|u^{\prime}\right\|^{2}+p \varepsilon\left\|u^{\prime}\right\|_{W^{\prime}}^{2}-\varepsilon \lambda_{1}^{2-\beta}\left\|A^{\frac{\beta}{2}-1} v^{\prime}\right\|_{W^{\prime}}^{2}-\frac{p-1}{2} \varepsilon\left\|A^{\frac{1}{2}} u\right\|_{W^{\prime}}^{2}-\varepsilon \frac{p-1}{2} \lambda_{1}^{2-\beta}\left\|A^{\frac{\beta}{2}} v\right\|_{W^{\prime}}^{2} \\
& +\varepsilon \lambda_{1}^{2-\beta} \alpha\left\langle A^{\beta-2} v, A^{\beta} u\right\rangle_{W^{\prime}}-p \varepsilon\left\langle u, u^{\prime}\right\rangle_{W^{\prime}}-p \varepsilon \alpha\left\langle u, A^{\beta} v\right\rangle_{W^{\prime}} \\
& +\rho \varepsilon\left\langle u^{\prime}, v^{\prime}\right\rangle_{W^{\prime}}-\rho \varepsilon\left\langle u^{\prime}, A^{-1} v^{\prime}\right\rangle_{W^{\prime}}-\rho \varepsilon\left\langle u^{\prime}, v\right\rangle_{W^{\prime}}
\end{aligned}
$$

Let us remark that

$$
\begin{aligned}
\varepsilon \lambda_{1}^{2-\beta} \alpha\left\langle A^{\beta-2} v, A^{\beta} u\right\rangle_{W^{\prime}} & \leq \varepsilon \lambda_{1}^{2-\beta}|\alpha|\left\|A^{\frac{\beta}{2}} v\right\|_{W^{\prime}}\left\|A^{\frac{3 \beta}{2}-2} u\right\|_{W^{\prime}} \\
& \leq \varepsilon \lambda_{1}^{2-\beta}|\alpha|\left\|A^{\frac{\beta}{2}} v\right\|_{W^{\prime}} \frac{1}{\lambda_{1}^{\frac{5-3 \beta}{2}}}\left\|A^{\frac{1}{2}} u\right\|_{W^{\prime}} \\
& \leq \varepsilon \lambda_{1}^{\frac{\beta-1}{2}}|\alpha|\left\|A^{\frac{\beta}{2}} v\right\|_{W^{\prime}}\left\|A^{\frac{1}{2}} u\right\|_{W^{\prime}}
\end{aligned}
$$

and that

$$
\begin{aligned}
-p \varepsilon \alpha\left\langle u, A^{\beta} v\right\rangle_{W^{\prime}} & \leq p \varepsilon|\alpha|\left\|A^{\frac{\beta}{2}} v\right\|_{W^{\prime}}\left\|A^{\frac{\beta}{2}} u\right\|_{W^{\prime}} \\
& \leq p \varepsilon \lambda_{1}^{\frac{\beta-1}{2}}|\alpha|\left\|A^{\frac{\beta}{2}} v\right\|_{W^{\prime}}\left\|A^{\frac{1}{2}} u\right\|_{W^{\prime}}
\end{aligned}
$$

Thus

$$
\begin{aligned}
& \varepsilon \lambda_{1}^{2-\beta} \alpha\left\langle A^{\beta-2} v, A^{\beta} u\right\rangle_{W^{\prime}}-p \varepsilon \alpha\left\langle u, A^{\beta} v\right\rangle_{W^{\prime}} \\
\leq & \varepsilon \lambda_{1}^{\frac{\beta-1}{2}}|\alpha|\left\|A^{\frac{\beta}{2}} v\right\|_{W^{\prime}}\left\|A^{\frac{1}{2}} u\right\|_{W^{\prime}}+p \varepsilon \lambda_{1}^{\frac{\beta-1}{2}}|\alpha|\left\|A^{\frac{\beta}{2}} v\right\|_{W^{\prime}}\left\|A^{\frac{1}{2}} u\right\|_{W^{\prime}} \\
\leq & \varepsilon \lambda_{1}^{\frac{\beta-1}{2}}|\alpha|(p+1)\left\|A^{\frac{\beta}{2}} v\right\|_{W^{\prime}}\left\|A^{\frac{1}{2}} u\right\|_{W^{\prime}} \\
\leq & \varepsilon \lambda_{1}^{\frac{\beta-1}{2}}(p+1) \frac{|\alpha|}{2}\left(\gamma\left\|A^{\frac{1}{2}} u\right\|_{W^{\prime}}^{2}+\frac{1}{\gamma}\left\|A^{\frac{\beta}{2}} v\right\|_{W^{\prime}}^{2}\right)
\end{aligned}
$$

where we choose $\gamma>0$ such that

$$
\delta:=\frac{p-1}{2}-\lambda_{1}^{\frac{\beta-1}{2}}(p+1) \frac{|\alpha|}{2} \gamma>0, \quad \zeta:=\frac{p-1}{2} \lambda_{1}^{2-\beta}-\lambda_{1}^{\frac{\beta-1}{2}}(p+1) \frac{|\alpha|}{2 \gamma}>0
$$

which is equivalent to

$$
\frac{\lambda_{1}^{\frac{\beta-1}{2}}(p+1)|\alpha|}{(p-1) \lambda_{1}^{2-\beta}}<\gamma<\frac{p-1}{\lambda_{1}^{\frac{\beta-1}{2}}(p+1)|\alpha|}
$$

This choice is possible provided that

$$
\left(\frac{p+1}{p-1}\right)^{2}<\frac{\lambda_{1}^{3-2 \beta}}{|\alpha|^{2}}
$$


Now we choose $p>1$ such that (3.10) is satisfied. Then we have

$$
\begin{aligned}
H_{\varepsilon}^{\prime}= & -\left\|u^{\prime}\right\|^{2}+p \varepsilon\left\|u^{\prime}\right\|_{W^{\prime}}^{2}-\varepsilon \lambda_{1}^{2-\beta}\left\|A^{\frac{\beta}{2}-1} v^{\prime}\right\|_{W^{\prime}}^{2}-\varepsilon \delta\left\|A^{\frac{1}{2}} u\right\|_{W^{\prime}}^{2}-\varepsilon \zeta\left\|A^{\frac{\beta}{2}} v\right\|_{W^{\prime}}^{2} \\
& -p \varepsilon\left\langle u, u^{\prime}\right\rangle_{W^{\prime}}+\rho \varepsilon\left\langle u^{\prime}, v^{\prime}\right\rangle_{W^{\prime}}-\rho \varepsilon\left\langle u^{\prime}, A^{-1} v^{\prime}\right\rangle_{W^{\prime}}-\rho \varepsilon\left\langle u^{\prime}, v\right\rangle_{W^{\prime}} .
\end{aligned}
$$

Now let us observe first that if one considers some $U \in W^{\prime}$ one has

$$
\|U\|_{W^{\prime}}^{2}=\left\langle U, A^{-2} U\right\rangle_{W^{\prime}, W}=\left\langle A^{-1 / 2} U, A^{-3 / 2} U\right\rangle_{V^{\prime}, V}=\left\|A^{-1 / 2} U\right\|_{V^{\prime}}^{2} .
$$

Second, since $u^{\prime} \in V$, one has $A u^{\prime} \in V^{\prime}$. Therefore according to (3.11) we have

$$
\left\|A u^{\prime}\right\|_{W^{\prime}}^{2}=\left\|A^{1 / 2} u^{\prime}\right\|_{V^{\prime}}^{2}
$$

Moreover $A^{1 / 2} u^{\prime} \in H$, thus, one has

$$
\left\|A u^{\prime}\right\|_{W^{\prime}}^{2}=\left\|A^{1 / 2} u^{\prime}\right\|_{V^{\prime}}^{2}=\left\langle A^{-1} A^{1 / 2} u^{\prime}, A^{1 / 2} u^{\prime}\right\rangle=\left\|u^{\prime}\right\|^{2} .
$$

According to (3.13), since

$$
\left\langle u^{\prime}, v^{\prime}\right\rangle_{W^{\prime}}=\left\langle A u^{\prime}, A^{-1} v^{\prime}\right\rangle_{W^{\prime}},
$$

we have

$$
\begin{aligned}
\left|\left\langle u^{\prime}, v^{\prime}\right\rangle_{W^{\prime}}\right| & \leq\left|\left\langle A u^{\prime}, A^{-1} v^{\prime}\right\rangle_{W^{\prime}}\right| \\
& \leq\left\|A u^{\prime}\right\|_{W^{\prime}}\left\|A^{-1} v^{\prime}\right\|_{W^{\prime}} \\
& =\left\|u^{\prime}\right\|\left\|A^{-1} v^{\prime}\right\|_{W^{\prime}} .
\end{aligned}
$$

Yet we have

$$
\left\|A^{-1} v^{\prime}\right\|_{W^{\prime}}^{2} \leq \frac{1}{\lambda_{1}^{\beta}}\left\langle A^{\beta} A^{-1} v^{\prime}, A^{-1} v^{\prime}\right\rangle_{W^{\prime}}=\frac{1}{\lambda_{1}^{\beta}}\left\|A^{\frac{\beta}{2}-1} v^{\prime}\right\|_{W^{\prime}}^{2} .
$$

Using Young's inequality, we find some constants $c_{1}, c_{2}, c_{3}, c_{4}>0$ such that

$$
\begin{aligned}
& -p\left\langle u, u^{\prime}\right\rangle_{W^{\prime}} \leq c_{1}\left\|u^{\prime}\right\|^{2}+\frac{\delta}{2}\left\|A^{\frac{1}{2}} u\right\|_{W^{\prime}}^{2} ; \quad(\delta \text { defined by }(3.9)) \\
& \rho\left\langle u^{\prime}, v^{\prime}\right\rangle_{W^{\prime}} \leq c_{2}\left\|u^{\prime}\right\|^{2}+\frac{\lambda_{1}^{2-\beta}}{3}\left\|A^{\frac{\beta}{2}-1} v^{\prime}\right\|_{W^{\prime}}^{2} \\
& \rho\left\langle u^{\prime}, A^{-1} v^{\prime}\right\rangle_{W^{\prime}} \leq c_{3}\left\|u^{\prime}\right\|^{2}+\frac{\lambda_{1}^{2-\beta}}{3}\left\|A^{\frac{\beta}{2}-1} v^{\prime}\right\|_{W^{\prime}}^{2} \\
& -\rho\left\langle u^{\prime}, v\right\rangle_{W^{\prime}} \leq c_{4}\left\|u^{\prime}\right\|^{2}+\frac{\zeta}{2}\left\|A^{\frac{\beta}{2}} v\right\|_{W^{\prime}}^{2} \quad(\zeta \text { defined by }(3.9))
\end{aligned}
$$

By choosing $\varepsilon$ small enough, we find a constant $\eta=\eta(p, \varepsilon)>0$ such that for all $t \geq 0$

$$
H_{\varepsilon}^{\prime} \leq-\eta\left(\left\|u^{\prime}\right\|^{2}+\left\|A^{\frac{\beta}{2}-1} v^{\prime}\right\|_{W^{\prime}}^{2}+\left\|A^{\frac{1}{2}} u\right\|_{W^{\prime}}^{2}+\left\|A^{\frac{\beta}{2}} v\right\|_{W^{\prime}}^{2}\right) .
$$


Let

$$
\begin{gathered}
\tilde{E}=\frac{1}{2}\left[\left\|A^{\frac{\beta}{2}-1} u^{\prime}(t)\right\|_{W^{\prime}}^{2}+\left\|A^{\frac{\beta}{2}-1} v^{\prime}(t)\right\|_{W^{\prime}}^{2}+\left\|A^{\frac{\beta-1}{2}} u(t)\right\|_{W^{\prime}}^{2}+\left\|A^{\frac{\beta}{2}} v(t)\right\|_{W^{\prime}}^{2}\right] \\
+\alpha\left\langle A^{2 \beta-2} v, u\right\rangle_{W^{\prime}}
\end{gathered}
$$

and

$$
K(t)=\left\|A^{\frac{\beta}{2}-1} u^{\prime}(t)\right\|_{W^{\prime}}^{2}+\left\|A^{\frac{\beta}{2}-1} v^{\prime}(t)\right\|_{W^{\prime}}^{2}+\left\|A^{\frac{\beta-1}{2}} u(t)\right\|_{W^{\prime}}^{2}+\left\|A^{\frac{\beta}{2}} v(t)\right\|_{W^{\prime}}^{2} .
$$

For all $t \geq 0$, we have

$$
\tilde{E}^{\prime}=-\left\|A^{\frac{\beta}{2}-1} u^{\prime}(t)\right\|_{W^{\prime}}^{2}
$$

Then $\tilde{E}$ is nonincreasing. Observe that

$$
\left|\alpha\left\langle A^{2 \beta-2} v, u\right\rangle_{W^{\prime}}\right| \leq \frac{|\alpha|}{\lambda_{1}^{\frac{3-2 \beta}{2}}}\left\|A^{\frac{\beta-1}{2}} u(t)\right\|_{W^{\prime}}\left\|A^{\frac{\beta}{2}} v(t)\right\|_{W^{\prime}},
$$

from which we deduce that

$$
\frac{\lambda_{1}^{\frac{3-2 \beta}{2}}-|\alpha|}{2 \lambda_{1}^{\frac{3-2 \beta}{2}}} K(t) \leq \tilde{E} \leq \frac{\lambda_{1}^{\frac{3-2 \beta}{2}}+|\alpha|}{2 \lambda_{1}^{\frac{3-2 \beta}{2}}} K(t) .
$$

Now since

$$
\left\|A^{\frac{\beta}{2}-1} u^{\prime}(t)\right\|_{W^{\prime}} \leq \frac{1}{\lambda_{1}^{2-\frac{\beta}{2}}}\left\|u^{\prime}\right\|, \quad\left\|A^{\frac{\beta-1}{2}} u(t)\right\|_{W^{\prime}} \leq \frac{1}{\lambda_{1}^{1-\frac{\beta}{2}}}\left\|A^{\frac{1}{2}} u\right\|_{W^{\prime}},
$$

then there exists a constant $\gamma>0$ such that for all $t \geq 0$

$$
H_{\varepsilon}^{\prime} \leq-\gamma K\left(u, v, u^{\prime}, v^{\prime}\right)
$$

From (3.15), assuming $\varepsilon$ possibly smaller in order to achieve positivity of the quadratic form $H_{\varepsilon}$, we get

$$
\int_{0}^{t} K\left(u(s), v(s), u^{\prime}(s), v^{\prime}(s)\right) d s \leq \frac{1}{\gamma} H_{\varepsilon}\left(u(0), v(0), u^{\prime}(0), v^{\prime}(0)\right) .
$$

Using inequality (3.14), we obtain

$$
\frac{2 \lambda_{1}^{\frac{3-2 \beta}{2}}}{\lambda_{1}^{\frac{3-2 \beta}{2}}+|\alpha|} \int_{0}^{t} \tilde{E}\left(u(s), v(s), u^{\prime}(s), v^{\prime}(s)\right) d s \leq \frac{1}{\gamma} H_{\varepsilon}\left(u(0), v(0), u^{\prime}(0), v^{\prime}(0)\right) .
$$


Now since $\tilde{E}$ is nonincreasing, it follows

$$
\tilde{E}\left(u(t), v(t), u^{\prime}(t), v^{\prime}(t)\right) \leq \frac{\lambda_{1}^{\frac{3-2 \beta}{2}}+|\alpha|}{2 \lambda_{1}^{\frac{3-2 \beta}{2}} \gamma} \frac{1}{t} H_{\varepsilon}\left(u(0), v(0), u^{\prime}(0), v^{\prime}(0)\right) .
$$

Using inequality (3.14) we get

$$
K\left(u(t), v(t), u^{\prime}(t), v^{\prime}(t)\right) \leq \frac{\lambda_{1}^{\frac{3-2 \beta}{2}}+|\alpha|}{\left(\lambda_{1}^{\frac{3-2 \beta}{2}}-|\alpha|\right) \gamma} \frac{1}{t} H_{\varepsilon}\left(u(0), v(0), u^{\prime}(0), v^{\prime}(0)\right) .
$$

Second case $: \beta \in\left(1, \frac{3}{2}\right]$. In this case $a=1-\beta$.

$$
\begin{aligned}
H_{\varepsilon}^{\prime}= & -\left\|u^{\prime}\right\|^{2}+p \varepsilon \lambda_{1}^{\beta-1}\left\|A^{\frac{1-\beta}{2}} u^{\prime}\right\|_{W^{\prime}}^{2}-\varepsilon \lambda_{1}^{2-\beta}\left\|A^{\frac{\beta}{2}-1} v^{\prime}\right\|_{W^{\prime}}^{2}-p \varepsilon \lambda_{1}^{\beta-1}\left\|A^{1-\frac{\beta}{2}} u\right\|_{W^{\prime}}^{2}-\varepsilon \frac{p-1}{2} \lambda_{1}^{2-\beta}\left\|A^{\frac{\beta}{2}} v\right\|_{W^{\prime}}^{2} \\
& +\rho \varepsilon \alpha\left\|A^{\frac{\beta-1}{2}} u\right\|_{W^{\prime}}^{2}+\varepsilon \lambda_{1}^{2-\beta} \alpha\left\langle A^{\beta-2} v, A^{\beta} u\right\rangle_{W^{\prime}}-p \varepsilon \lambda_{1}^{\beta-1}\left\langle A^{1-\beta} u, u^{\prime}\right\rangle \\
& -p \varepsilon \lambda_{1}^{\beta-1} \alpha\left\langle A^{1-\beta} u, A^{\beta} v\right\rangle_{W^{\prime}}+\rho \varepsilon\left\langle u^{\prime}, v^{\prime}\right\rangle_{W^{\prime}}-\rho \varepsilon\left\langle u^{\prime}, A^{-1} v^{\prime}\right\rangle_{W^{\prime}}-\rho \varepsilon\left\langle u^{\prime}, v\right\rangle_{W^{\prime}} .
\end{aligned}
$$

Now since

$$
\left\|A^{\frac{\beta-1}{2}} u\right\|_{W^{\prime}}^{2} \leq \frac{1}{\lambda_{1}^{3-2 \beta}}\left\langle A^{2-\beta} u, u\right\rangle_{W^{\prime}}=\frac{1}{\lambda_{1}^{3-2 \beta}}\left\|A^{1-\frac{\beta}{2}} u\right\|_{W^{\prime}}^{2},
$$

we get

$$
\begin{aligned}
H_{\varepsilon}^{\prime} \leq & -\left\|u^{\prime}\right\|^{2}+p \varepsilon \lambda_{1}^{\beta-1}\left\|A^{\frac{1-\beta}{2}} u^{\prime}\right\|_{W^{\prime}}^{2}-\varepsilon \lambda_{1}^{2-\beta}\left\|A^{\frac{\beta}{2}-1} v^{\prime}\right\|_{W^{\prime}}^{2}-\frac{p-1}{2} \varepsilon \lambda_{1}^{\beta-1}\left\|A^{1-\frac{\beta}{2}} u\right\|_{W^{\prime}}^{2} \\
& -\varepsilon \frac{p-1}{2} \lambda_{1}^{2-\beta}\left\|A^{\frac{\beta}{2}} v\right\|_{W^{\prime}}^{2}+\varepsilon \lambda_{1}^{2-\beta} \alpha\left\langle A^{\beta-2} v, A^{\beta} u\right\rangle_{W^{\prime}}-p \varepsilon \lambda_{1}^{\beta-1}\left\langle A^{1-\beta} u, u^{\prime}\right\rangle \\
& -p \varepsilon \lambda_{1}^{\beta-1} \alpha\left\langle A^{1-\beta} u, A^{\beta} v\right\rangle_{W^{\prime}}+\rho \varepsilon\left\langle u^{\prime}, v^{\prime}\right\rangle_{W^{\prime}}-\rho \varepsilon\left\langle u^{\prime}, A^{-1} v^{\prime}\right\rangle_{W^{\prime}}-\rho \varepsilon\left\langle u^{\prime}, v\right\rangle_{W^{\prime}}
\end{aligned}
$$

Let us remark that

$$
\begin{aligned}
\varepsilon \lambda_{1}^{2-\beta} \alpha\left\langle A^{\beta-2} v, A^{\beta} u\right\rangle_{W^{\prime}} & \leq \varepsilon \lambda_{1}^{2-\beta}|\alpha|\left\|A^{\frac{\beta}{2}} v\right\|_{W^{\prime}}\left\|A^{\frac{3 \beta}{2}-2} u\right\|_{W^{\prime}} \\
& \leq \varepsilon \lambda_{1}^{2-\beta}|\alpha|\left\|A^{\frac{\beta}{2}} v\right\|_{W^{\prime}} \frac{1}{\lambda_{1}^{3-2 \beta}}\left\|A^{1-\frac{\beta}{2}} u\right\|_{W^{\prime}} \\
& \leq \varepsilon \lambda_{1}^{\beta-1}|\alpha|\left\|A^{\frac{\beta}{2}} v\right\|_{W^{\prime}}\left\|A^{1-\frac{\beta}{2}} u\right\|_{W^{\prime}}
\end{aligned}
$$

and that

$$
-p \varepsilon \lambda_{1}^{\beta-1} \alpha\left\langle A^{1-\beta} u, A^{\beta} v\right\rangle_{W^{\prime}} \leq p \varepsilon \lambda_{1}^{\beta-1}|\alpha|\left\|A^{\frac{\beta}{2}} v\right\|_{W^{\prime}}\left\|A^{1-\frac{\beta}{2}} u\right\|_{W^{\prime}},
$$

we therefore get

$$
\begin{aligned}
& \varepsilon \lambda_{1}^{2-\beta} \alpha\left\langle A^{\beta-2} v, A^{\beta} u\right\rangle_{W^{\prime}}-p \varepsilon \lambda_{1}^{\beta-1} \alpha\left\langle A^{1-\beta} u, A^{\beta} v\right\rangle_{W^{\prime}} \\
\leq & \varepsilon \lambda_{1}^{\beta-1}|\alpha|\left\|A^{\frac{\beta}{2}} v\right\|_{W^{\prime}}\left\|A^{1-\frac{\beta}{2}} u\right\|_{W^{\prime}}+p \varepsilon \lambda_{1}^{\beta-1}|\alpha|\left\|A^{\frac{\beta}{2}} v\right\|_{W^{\prime}}\left\|A^{1-\frac{\beta}{2}} u\right\|_{W^{\prime}} \\
= & \varepsilon(p+1) \lambda_{1}^{\beta-1}|\alpha|\left\|A^{\frac{\beta}{2}} v\right\|_{W^{\prime}}\left\|A^{1-\frac{\beta}{2}} u\right\|_{W^{\prime}}
\end{aligned}
$$




$$
\leq \varepsilon \lambda_{1}^{\beta-1}(p+1) \frac{|\alpha|}{2}\left(\gamma\left\|A^{1-\frac{\beta}{2}} u\right\|_{W^{\prime}}^{2}+\frac{1}{\gamma}\left\|A^{\frac{\beta}{2}} v\right\|_{W^{\prime}}^{2}\right)
$$

where we choose $\gamma>0$ such that

$$
\delta:=\frac{p-1}{2} \lambda_{1}^{\beta-1}-\lambda_{1}^{\beta-1}(p+1) \frac{|\alpha|}{2} \gamma>0, \quad \zeta:=\frac{p-1}{2} \lambda_{1}^{2-\beta}-\lambda_{1}^{\beta-1}(p+1) \frac{|\alpha|}{2 \gamma}>0
$$

which is equivalent to

$$
\frac{\lambda_{1}^{\beta-1}(p+1)|\alpha|}{(p-1) \lambda_{1}^{2-\beta}}<\gamma<\frac{p-1}{(p+1)|\alpha|} .
$$

This choice is possible provided that

$$
\left(\frac{p+1}{p-1}\right)^{2}<\frac{\lambda_{1}^{3-2 \beta}}{|\alpha|^{2}}
$$

We choose $p>1$ such that (3.16) is satisfied. Then we have

$$
\begin{aligned}
H_{\varepsilon}^{\prime} \leq & -\left\|u^{\prime}\right\|^{2}+p \varepsilon \lambda_{1}^{\beta-1}\left\|A^{\frac{1-\beta}{2}} u^{\prime}\right\|_{W^{\prime}}^{2}-\varepsilon \lambda_{1}^{2-\beta}\left\|A^{\frac{\beta}{2}-1} v^{\prime}\right\|_{W^{\prime}}^{2}-\varepsilon \delta\left\|A^{1-\frac{\beta}{2}} u\right\|_{W^{\prime}}^{2}-\varepsilon \zeta\left\|A^{\frac{\beta}{2}} v\right\|_{W^{\prime}}^{2} \\
& -p \varepsilon \lambda_{1}^{\beta-1}\left\langle A^{1-\beta} u, u^{\prime}\right\rangle+\rho \varepsilon\left\langle u^{\prime}, v^{\prime}\right\rangle_{W^{\prime}}-\rho \varepsilon\left\langle u^{\prime}, A^{-1} v^{\prime}\right\rangle_{W^{\prime}}-\rho \varepsilon\left\langle u^{\prime}, v\right\rangle_{W^{\prime}} .
\end{aligned}
$$

By use of Young's inequality we deduce that there are $c_{1}, c_{2}, c_{3}, c_{4}>0$ such that

$$
\begin{aligned}
& -p \lambda_{1}^{\beta-1}\left\langle A^{1-\beta} u, u^{\prime}\right\rangle \leq c_{1}\left\|u^{\prime}\right\|^{2}+\frac{\delta}{2}\left\|A^{1-\frac{\beta}{2}} u\right\|_{W^{\prime}}^{2}, \\
& \rho\left\langle u^{\prime}, v^{\prime}\right\rangle_{W^{\prime}} \leq c_{2}\left\|u^{\prime}\right\|^{2}+\frac{\lambda_{1}^{2-\beta}}{3}\left\|A^{\frac{\beta}{2}-1} v^{\prime}\right\|_{W^{\prime}}^{2}, \\
& \rho \varepsilon\left\langle u^{\prime}, A^{-1} v^{\prime}\right\rangle_{W^{\prime}} \leq c_{3}\left\|u^{\prime}\right\|^{2}+\frac{\lambda_{1}^{2-\beta}}{3}\left\|A^{\frac{\beta}{2}-1} v^{\prime}\right\|_{W^{\prime}}^{2}, \\
& -\rho\left\langle u^{\prime}, v\right\rangle_{W^{\prime}} \leq c_{4}\left\|u^{\prime}\right\|^{2}+\frac{\zeta}{2}\left\|A^{\frac{\beta}{2}} v\right\|_{W^{\prime}}^{2} .
\end{aligned}
$$

By choosing $\varepsilon$ small enough, we find a constant $\eta=\eta(p, \varepsilon)>0$ such that for all $t \geq 0$

$$
H_{\varepsilon}^{\prime} \leq-\eta\left(\left\|u^{\prime}\right\|^{2}+\left\|A^{\frac{\beta}{2}-1} v^{\prime}\right\|_{W^{\prime}}^{2}+\left\|A^{1-\frac{\beta}{2}} u\right\|_{W^{\prime}}^{2}+\left\|A^{\frac{\beta}{2}} v\right\|_{W^{\prime}}^{2}\right)
$$

Let

$$
\tilde{E}=\frac{1}{2}\left[\left\|A^{\frac{-\beta}{2}} u^{\prime}(t)\right\|_{W^{\prime}}^{2}+\left\|A^{\frac{-\beta}{2}} v^{\prime}(t)\right\|_{W^{\prime}}^{2}+\left\|A^{\frac{1-\beta}{2}} u(t)\right\|_{W^{\prime}}^{2}+\left\|A^{1-\frac{\beta}{2}} v(t)\right\|_{W^{\prime}}^{2}\right]+\alpha\langle v, u\rangle_{W^{\prime}}
$$

and

$$
K(t)=\left\|A^{\frac{-\beta}{2}} u^{\prime}(t)\right\|_{W^{\prime}}^{2}+\left\|A^{\frac{-\beta}{2}} v^{\prime}(t)\right\|_{W^{\prime}}^{2}+\left\|A^{\frac{1-\beta}{2}} u(t)\right\|_{W^{\prime}}^{2}+\left\|A^{1-\frac{\beta}{2}} v(t)\right\|_{W^{\prime}}^{2}
$$


For all $t \geq 0$, we have

$$
\tilde{E}^{\prime}=-\left\|A^{-\frac{\beta}{2}} u^{\prime}(t)\right\|_{W^{\prime}}^{2}
$$

Then $\tilde{E}$ is nonincreasing.

Since

$$
\left|\alpha\langle v, u\rangle_{W^{\prime}}\right| \leq \frac{|\alpha|}{\lambda_{1}^{\frac{3-2 \beta}{2}}}\left\|A^{\frac{1-\beta}{2}} u(t)\right\|_{W^{\prime}}\left\|A^{1-\frac{\beta}{2}} v(t)\right\|_{W^{\prime}}
$$

then we get

$$
\frac{\lambda_{1}^{\frac{3-2 \beta}{2}}-|\alpha|}{2 \lambda_{1}^{\frac{3-2 \beta}{2}}} K(t) \leq \tilde{E} \leq \frac{\lambda_{1}^{\frac{3-2 \beta}{2}}+|\alpha|}{2 \lambda_{1}^{\frac{3-2 \beta}{2}}} K(t) .
$$

Now since

$$
\left\|A^{-\frac{\beta}{2}} v^{\prime}(t)\right\|_{W^{\prime}} \leq \frac{1}{\lambda_{1}^{\beta-1}}\left\|A^{\frac{\beta}{2}-1} v^{\prime}\right\|_{W^{\prime}}, \quad\left\|A^{\frac{1-\beta}{2}} u(t)\right\|_{W^{\prime}} \leq \frac{1}{\lambda_{1}^{\frac{1}{2}}}\left\|A^{1-\frac{\beta}{2}} u\right\|_{W^{\prime}}
$$

then there exists a constant $\gamma>0$ such that for all $t \geq 0$

$$
H_{\varepsilon}^{\prime} \leq-\gamma K\left(u, v, u^{\prime}, v^{\prime}\right)
$$

From (3.18), assuming $\varepsilon$ possibly smaller in order to achieve positivity of the quadratic form $H_{\varepsilon}$, we get

$$
\int_{0}^{t} K\left(u(s), v(s), u^{\prime}(s), v^{\prime}(s)\right) d s \leq \frac{1}{\gamma} H_{\varepsilon}\left(u(0), v(0), u^{\prime}(0), v^{\prime}(0)\right) .
$$

Using inequality (3.17), we obtain

$$
\frac{2 \lambda_{1}^{\frac{3-2 \beta}{2}}}{\lambda_{1}^{\frac{3-2 \beta}{2}}+|\alpha|} \int_{0}^{t} \tilde{E}\left(u(s), v(s), u^{\prime}(s), v^{\prime}(s)\right) d s \leq \frac{1}{\gamma} H_{\varepsilon}\left(u(0), v(0), u^{\prime}(0), v^{\prime}(0)\right) .
$$

Now since $\tilde{E}$ is nonincreasing, it follows

$$
\tilde{E}\left(u(t), v(t), u^{\prime}(t), v^{\prime}(t)\right) \leq \frac{\lambda_{1}^{\frac{3-2 \beta}{2}}+|\alpha|}{2 \lambda_{1}^{\frac{3-2 \beta}{2}} \gamma} \frac{1}{t} H_{\varepsilon}\left(u(0), v(0), u^{\prime}(0), v^{\prime}(0)\right) .
$$

Using inequality (3.17) we get

$$
K\left(u(t), v(t), u^{\prime}(t), v^{\prime}(t)\right) \leq \frac{\lambda_{1}^{\frac{3-2 \beta}{2}}+|\alpha|}{\left(\lambda_{1}^{\frac{3-2 \beta}{2}}-|\alpha|\right) \gamma} \frac{1}{t} H_{\varepsilon}\left(u(0), v(0), u^{\prime}(0), v^{\prime}(0)\right) .
$$




\section{EXAMPLES}

This section is devoted to giving examples of operators to which Theorem 3.3 applies.

Example 4.1. The first case that we consider is when $H=L^{2}(\Omega)$ and

$$
A u=-\sum_{i, j=1}^{N} \frac{\partial}{\partial x_{i}}\left(a_{i j} \frac{\partial}{\partial x_{j}} u\right),
$$

where the coefficients $a_{i, j} \in C^{1}(\bar{\Omega})$ satisfy

$$
a_{i j}=a_{j i}, \forall i, j,
$$

and the matrix $\left(a_{i, j}\right)$ is uniformly coercive on $\bar{\Omega}$. With these assumptions we have $D(A)=H^{2}(\Omega) \cap H_{0}^{1}(\Omega)$.

Example 4.2. The second example considered is $H=L^{2}(\Omega), D(A)=\left\{u \in H^{2}(\Omega), \frac{\partial u}{\partial n}=0\right.$ on $\left.\partial \Omega\right\}$, and

$$
A u=-\Delta u+\rho_{1} u
$$

where $\rho_{1}>0$

Here we can, as in [4] consider the case where

$$
D\left(A_{2}\right)=D\left(A^{2}\right), A_{2} u=\Delta^{2} u+\rho_{2} u,
$$

where $\rho_{2}>0$.

Example 4.3. Let us remark that due to remark 3.5 and the Poincaré inequality, our result applies to the case when $A_{1}=A$ is as in example 1 and $A_{2}=A_{1}^{2}+\zeta A_{1}$ for any $\zeta>0$.

\section{REFERENCES}

[1] M. Afilal and F. Ammar Khodja, Stability of coupled second order equations. Comput. Appl. Math. 19 (2000) $91-107$.

[2] F. Abdallah, Y. Chitour, M. Ghader and A. Wehbe, Optimal indirect stability of a weakly damped elastic abstract system of second order equations coupled by velocities. Comm. Pure Appl. Anal. 18 (2019) 2789-2818.

[3] F. Alabau-Boussouira, Indirect boundary stabilization of weakly coupled hyperbolic systems. SIAM J. Control. Optim. 41 (2002) 511-541.

[4] F. Alabau, P. Cannarsa and V. Komornik, Indirect internal stabilization of weakly coupled evolution equations. J. Evol. Equ. 2 (2002) $127-150$

[5] F. Alabau-Boussouira and M. Léautaud, Indirect stabilization of locally coupled wave-type systems. ESAIM: COCV 18 (2011) $548-582$.

[6] K. Ammari, E.M.A. Benhassi, S. Boulite and L. Maniar, Exponential energy decay of some coupled second order systems. Semigroup Forum 86 (2012) 362-382.

[7] K. Ammari and M. Mehrenberger, Stabilization of coupled systems. Acta Math. Hungar. 123 (2009) 1-10.

[8] A. Haraux and M.A. Jendoubi, The convergence problem for dissipative autonomous systems - classical methods and recent advances. SpringerBriefs in Mathematics. Springer, Cham (2015).

[9] A. Haraux and M.A. Jendoubi, A Liapunov function approach to the stabilization of second order coupled systems. North-West. Eur. J. Math. 2 (2016) 121-144.

[10] V. Komornik, Exact Controllability and Stabilization: The Multiplier Method. Exact Controllability and Stabilization : The Multiplier Method. Wiley-Masson Series Research in Applied Mathematics, Wiley (1995).

[11] P. Loreti and B.P. Rao, Optimal energy decay rate for partially damped systems by spectral compensation. SIAM J. Control. Optim. 45 (2006) 1612-1632.

[12] S. Marx, Y. Chitour and C. Prieur, Stability analysis of dissipative systems subject to nonlinear damping via Lyapunov techniques. ArXiv preprint arXiv:1808.05370 (2018) 